\title{
An Unusual Case of Colon Perforation With Multiple Transmural Ulcers After Use of Polmacoxib and Everolimus in a Metastatic Breast Cancer Patient
}

\author{
In-Gyu Song, Kyung Uk Jung, Hyung Ook Kim, Hungdai Kim, Ho-Kyung Chun \\ Department of Surgery, Kangbuk Samsung Hospital, Sungkyunkwan University School of Medicine, Seoul, Korea
}

Everolimus (Afinitor) is an inhibitor of mammalian target of rapamycin. Polmacoxib (Acelex) is a nonsteroidal anti-inflammatory drug that belongs to the cyclooxygenase-2 (COX-2) inhibitor family and is mainly used for treatment of arthritis. Intestinal perforation has not been reported previously as a complication of everolimus, and perforation of the lower intestinal tract caused by a selective COX-2 inhibitor is extremely rare. We present here a case of colon perforation that occurred after use of polmacoxib in a metastatic breast cancer patient who had been treated with everolimus for the preceding six months.

Keywords: Colon perforation; Everolimus; mTOR inhibitor; Polmacoxib, Nonsteroidal anti-inflammatory agents

\section{INTRODUCTION}

Colon perforation is one of the most serious adverse events of anticancer therapy. However, there are few reports of colon perforation related to treatment of breast cancer. Everolimus (Afinitor) is an orally available inhibitor of mammalian target of rapamycin (mTOR) and has been approved in combination with exemestane for treatment of hormone-receptor-positive breast cancer after failure of treatment with nonsteroidal aromatase inhibitors [1]. Everolimus is generally well tolerated, and its most common side effects include stomatitis, rash, fatigue, hyperglycemia, hyperlipidemia, and myelosuppression. Most of these side effects are mild and resolve with interruption or reduction of dosage [2]. Intestinal perforation has not been reported previously as a complication of everolimus.

Received: May 30, 2019 - Revised: Aug 11, 2019 • Accepted: Aug 17, 2019 Correspondence to: Kyung Uk Jung, M.D.

Department of Surgery, Kangbuk Samsung Hospital, Sungkyunkwan University School of Medicine, 29 Saemunan-ro, Jongno-gu, Seoul 03181, Korea

Tel: +82-2-2001-8408, Fax: 82-2-2001-8306

E-mail: sahelgrean@gmail.com

ORCID: https://orcid.org/0000-0002-8844-3724

(C) 2021 The Korean Society of Coloproctology

This is an open-access article distributed under the terms of the Creative Commons Attribution NonCommercial License (https://creativecommons.org/licenses/by-nc/4.0) which permits unrestricted noncommercial use, distribution, and reproduction in any medium, provided the original work is properly cited.
Polmacoxib (Acelex) is a nonsteroidal anti-inflammatory drug (NSAID) used to treat osteoarthritis. Unlike other NSAIDs, polmacoxib has a dual mode of action: inhibition of cyclooxygenase-2 (COX-2) and binding to carbonic anhydrase with high affinity. Therefore, polmacoxib has potential for use as a pain reliever with reduced gastrointestinal side effects compared with traditional NSAIDs for arthritis [3]. Lower intestine perforation caused by selective COX-2 inhibitors has been reported, but its incidence is extremely low. We present here a case of colon perforation that occurred after use of polmacoxib in a patient with metastatic breast cancer who had been treated with everolimus for the preceding 6 months.

\section{CASE REPORT}

A 66-year-old Korean woman who had been hospitalized in the orthopedic department developed acute-onset whole-abdominal pain following hematochezia. She had undergone a modified radical mastectomy with axillary lymph node dissection for right breast cancer (pT2N1M0, stage IIB) 5 years previous. The patient had received six cycles of adjuvant chemotherapy with CMF (cyclophosphamide $600 \mathrm{mg} / \mathrm{m}^{2}$, methotrexate $40 \mathrm{mg} / \mathrm{m}^{2}$, and fluorouracil $600 \mathrm{mg} / \mathrm{m}^{2}$ ) regimen one month after breast surgery. Four years after surgery, bone metastasis was detected on the T8 vertebra. Three months after detection of this bone metastasis, the chemotherapy regimen was changed to everolimus (10 mg/day) 
Volume 37, Number 2, 2021

with an aromatase inhibitor. Seven months after changing this chemotherapy regimen, the patient received an arthrotomy of the left wrist because of seronegative rheumatoid arthritis and then began polmacoxib medication. Seventeen days after starting polmacoxib, the patient complained of acute-onset whole-abdominal pain following hematochezia.

The patient had no other underlying disease except breast cancer and seronegative rheumatoid arthritis. She had not received any radiotherapy or steroids and had no past history of chronic intestinal ailment. On physical examination, the patient appeared acutely ill. Her body temperature was $37.5^{\circ} \mathrm{C}$, pulse rate was 87 beats per minute, and blood pressure was 111/68 mmHg. Her abdomen was rigid and exhibited whole-abdomen tenderness and rebound tenderness. The maximal point of tenderness was the left upper quadrant. Rectal examination showed no active bleeding or rectal mass. Laboratory tests showed increased leukocyte count $12.44 \times 10^{3} / \mathrm{mm}^{3}$ with neutrophilia, decreased hemoglobin count $9.9 \mathrm{~g} / \mathrm{dL}$, hematocrit level $29.8 \%$, and platelet count $332 \times$ $10^{3} / \mathrm{mm}^{3}$. Prothrombin time and international normalized ratio were prolonged at 14.5 and 1.36 seconds, respectively. An abdominopelvic computed tomography (APCT) scan showed multiple wall defects in the descending colon with perilesional fat infiltration and pneumoperitoneum, suggesting multiple perforations of the descending colon (Fig. 1).

Emergency laparoscopic exploration was performed. Intraoperative findings included diffuse inflammation and multiple abscess pockets in the mesocolon, severely inflamed left colon, and a gross perforation in the proximal descending colon measuring 2 $\mathrm{cm}$ that was connected to the largest abscess pocket. A left hemicolectomy with abscess drainage and irrigation of the peritoneal cavity was performed using hand-assisted laparoscopy. The severely inflamed left colon and perforation site were resected, and a transverse sigmoid anastomosis was created. The specimen obtained during the operation showed multiple transmural ulcer-

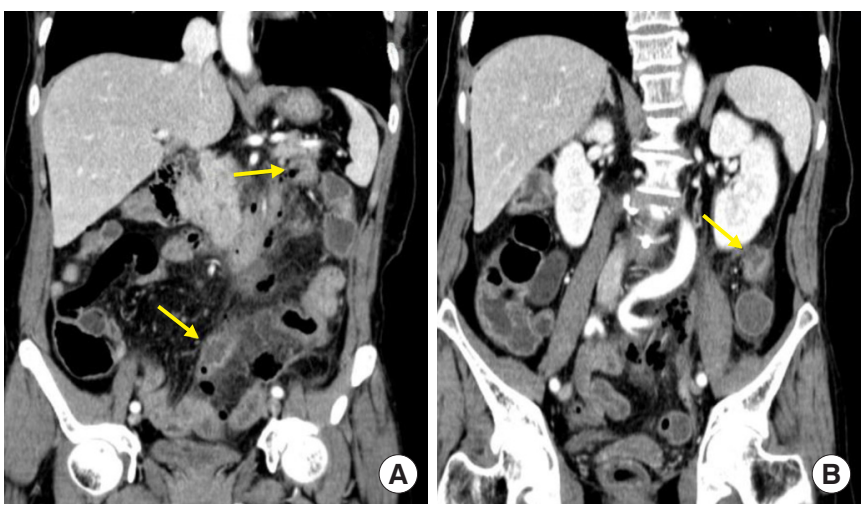

Fig. 1. Abdominopelvic computed tomography scan showed multiple wall defects of the descending colon with perilesional fat infiltration (A, yellow arrow) and pneumoperitoneum (B, perihapatic free air), suggesting multiple perforations of the descending colon. ations adjacent to the gross perforation site (Fig. 2). There was no evidence of underlying gastrointestinal disease such as diverticulitis or inflammatory bowel disease. Pathological evaluation of the specimen showed chronic active transmural inflammation, vascular congestion, and acute serositis (Fig. 3).

After operation, the patient was treated with intravenous antibiotics for peritonitis, and all previous oral medications, including everolimus and polmacoxib, were discontinued. The patient's abdominal pain improved on postoperative day 1 . However, on postoperative day 3, fresh red blood hematochezia without abdominal pain started. Under suspicion of intraluminal bleeding at the anastomosis site, emergency sigmoidoscopy was performed, which showed that the anastomosis was clean and patent and multiple geographic ulcers existed in the remnant colon wall (Fig. 4). Although the range of ulceration involved the whole colon, there was no sign of additional perforation, and we chose not to return the patient to the operating room immediately.

Considering the possibility of total colectomy, we observed this patient closely. On postoperative day 5 , the hematochezia stopped spontaneously after conservative management. On postoperative day 8, follow-up APCT showed diffuse edematous wall thickening from the distal transverse colon to the rectosigmoid colon. Loculated fluid appeared in the lower abdomen, and percutaneous drainage was performed. There was no evidence of newly de-

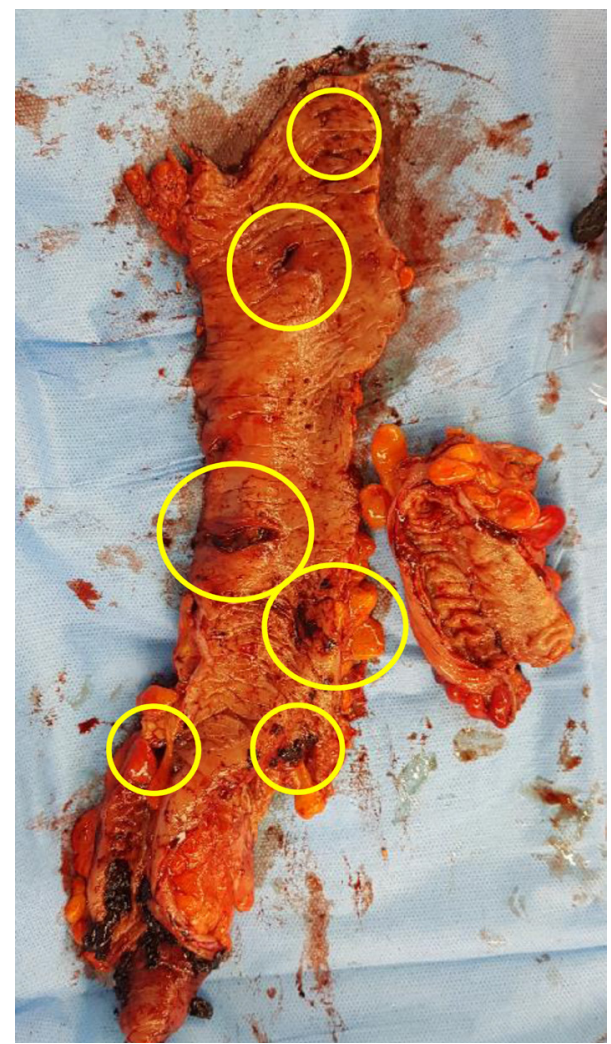

Fig. 2. Multiple ulcerations (yellow circles) in the delivered specimen. 


\section{Annals of $\quad$ An Unusual Case of Colon Perforation With Multiple Transmural Ulcers After Use of Polmacoxib and}

$\begin{array}{rlr}\text { Annals of } & \text { Everolimus in a Metaproctology } & \text { In-Gyu Song, et al. }\end{array}$

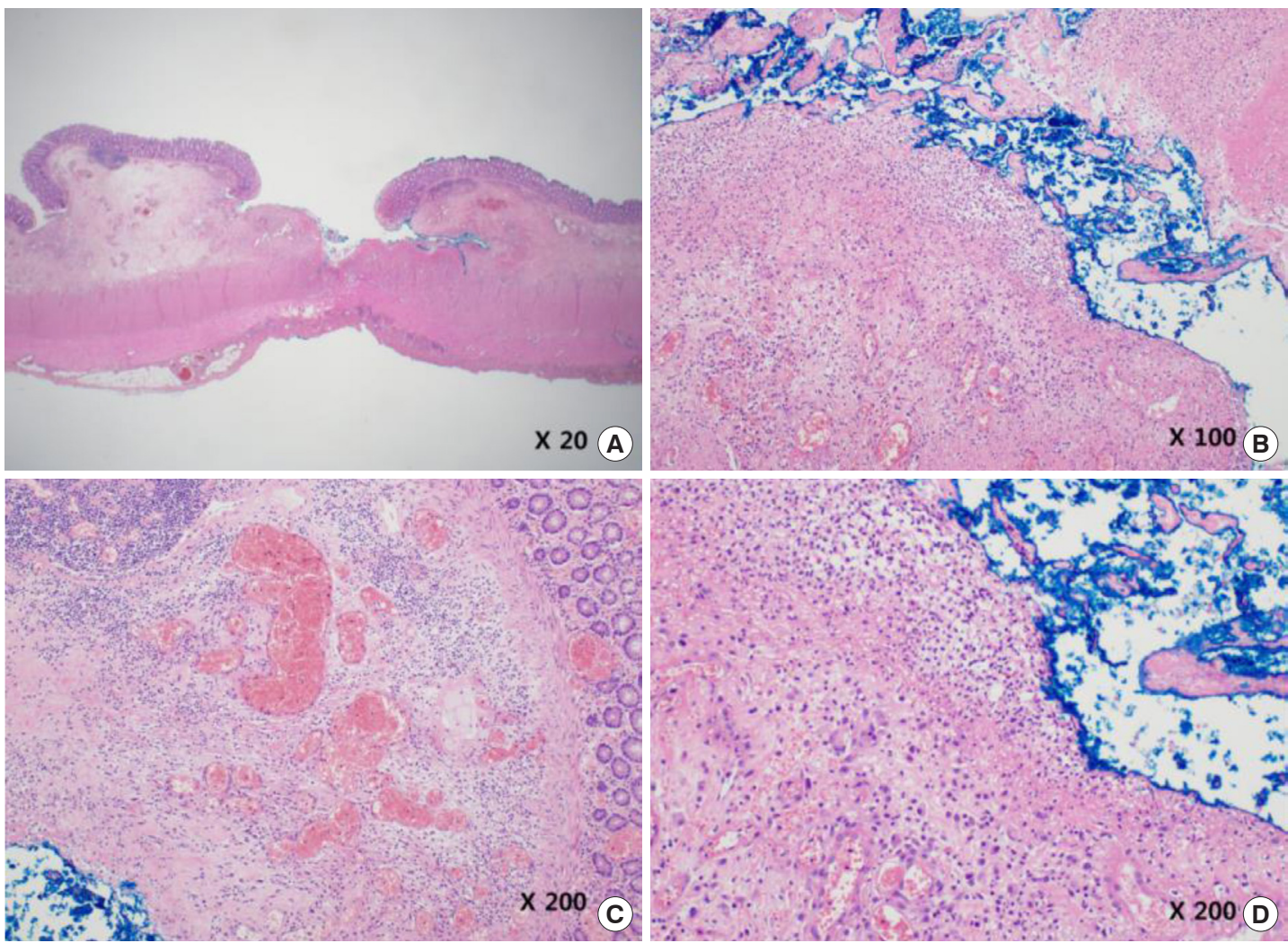

Fig. 3. Pathologic evaluation of the specimen using H\&E stain, showing chronic active transmural inflammation (A, B, D), vascular congestion (C), and acute serositis (A).
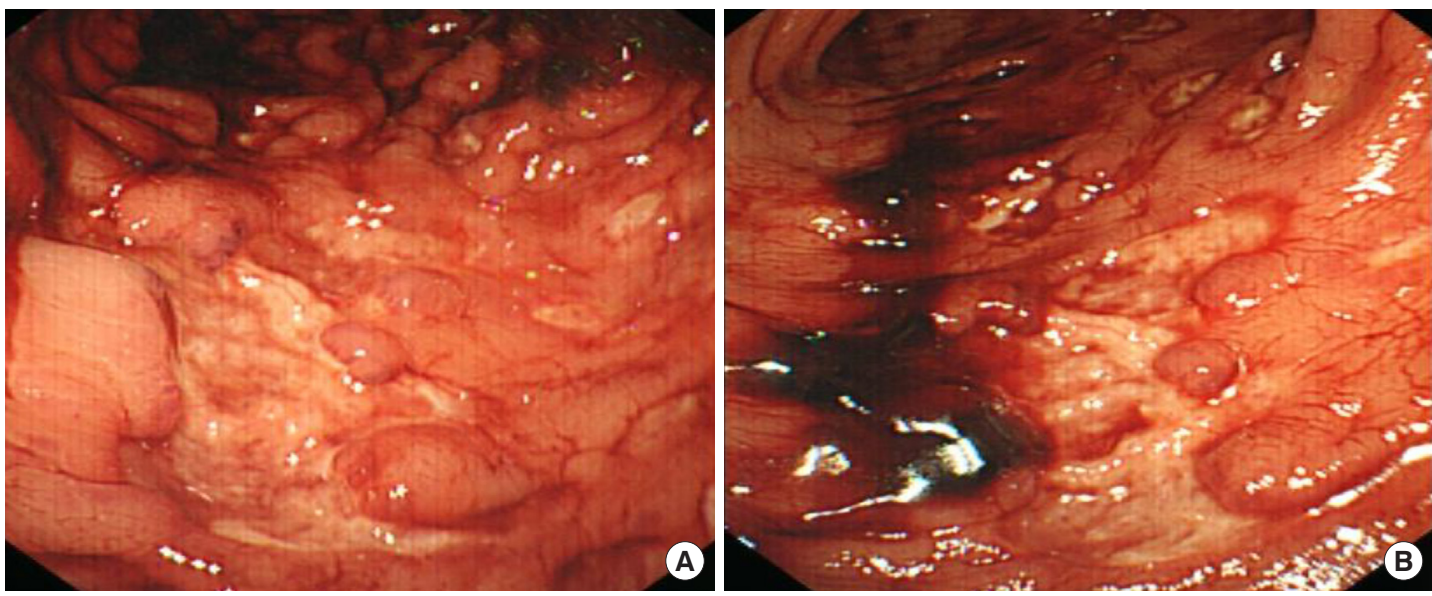

Fig. 4. Emergency sigmoidoscopy showed multiple geographic ulcers (A) in the remnant colonic wall (B).

veloped colon perforation. The patient started an oral diet on postoperative day 13 and was discharged home on postoperative day 34. Colonoscopy was performed 6 months after the operation and showed that the colon ulceration had self-resolved, and the remainder of the colon was normal (Fig. 5).

This study was approved by the Institutional Review Board of the Kangbuk Samsung Hospital (No. 2019-05-024). Written informed consent was waived.

\section{DISCUSSION}

Everolimus in combination with oral exemestane was approved 


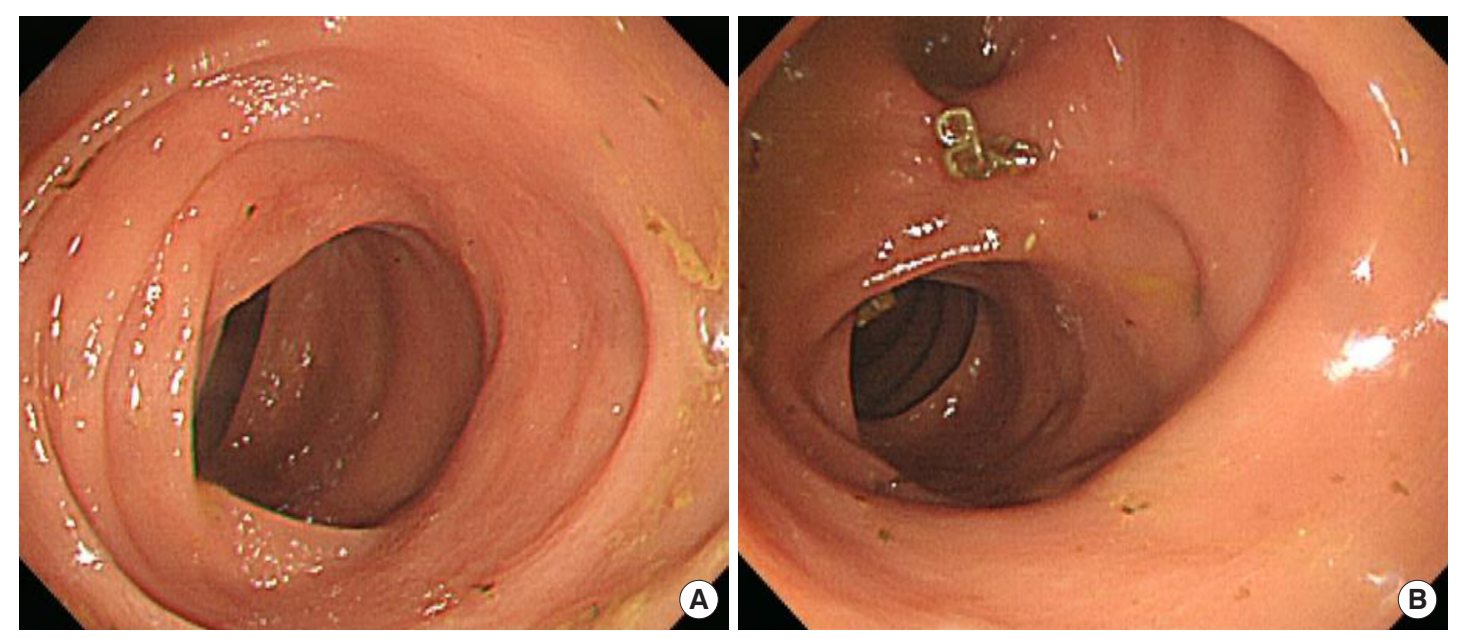

Fig. 5. Ulcerations of the colon wall self-resolved, and remnant colonic mucosa was normal in colonoscopy 6 months after surgery. (A) Sigmoid colon which was severely ulcerated in the past, (B) anastomosis site.

for treatment of postmenopausal patients with hormone-receptor-positive, human epidermal growth factor-2-negative advanced breast cancer that recurs or progresses during or after nonsteroidal aromatase inhibitor treatment [4]. The efficacy of everolimus has been reported, but serious adverse events have also been reported, including stomatitis, rash, fatigue, hyperglycemia, hyperlipidemia, and myelosuppression. There have been no reports of intestinal perforation caused by everolimus, although there are a few reports of intestinal perforation caused by other breast chemotherapeutic agents. Some of the perforation cases related to breast cancer treatment may have been caused by metastasis [5].

Colon perforation caused by mTOR inhibitors is rare, has unknown etiology, and may involve a complex mechanism. In 2006, Hugl et al. [6] reported colon perforation associated with leukocytoclastic vasculitis (LCV) caused by sirolimus toxicity. In that study, multiple ulcers were noted in various places throughout the colon. Histology revealed nonspecific ulceration with atypical inflammatory changes within the wall and signs of vasculitis. The resected specimen obtained from our patient (Figs. 2 and 3) and the results of colonoscopy on postoperative day 5 (Fig. 4) are similar to the findings of Hugl et al. [6].

LCV is a type of necrotizing vasculitis with segmental areas of transmural infiltration and disruption of the vessel architecture by neutrophils, accompanied by fibrinoid necrosis caused by autoimmune disease, infection, or certain drugs [7]. LCV involves mainly mesenteric blood vessels and causes ischemic ulcers within the dependent colon segments, and colonoscopy biopsy cannot identify this process. LCV has been described primarily in the skin, but many patients present with systemic manifestations involving joints, kidneys, and the gastrointestinal tract. Sirolimusassociated cutaneous LCV has been reported after kidney-pancreas and lung transplantation and in children [8-10]. Everolimus is a derivate of sirolimus, and we suggest that a similar mecha- nism as that causing colon perforation might be involved.

The selective COX-2 inhibitor polmacoxib has been approved in Korea only for treatment of arthritis; this drug has similar efficacy and cardiovascular safety to celecoxib, although no largescale safety studies have been performed [11]. Although the most frequent places for gastrointestinal toxicity of conventional NSAIDs are the stomach and proximal duodenum, toxicity is not necessarily confined to these areas but may also extend to the rest of the gastrointestinal tract, mid- to distal duodenum, small bowel, colon, and rectum. Selective COX-2 inhibitors have reduced risk of gastrointestinal toxicity compared with conventional NSAIDs, but risk is not absent [12].

We could not determine which agent-everolimus or polmacoxib-was responsible for colon perforation in this patient. Most cases of NSAID-induced colopathy present a single ulcerative lesion, colon stricture, and diaphragm-like stricture. However, in our case, there were multiple perforated lesions and transmural ulcers. There were no strictures or diaphragm-like lesions upon colonoscopy. As a result, we thought colon perforation was more likely caused by LCV. Of course, the possibility of colon perforation caused by rheumatoid arthritis and NSAIDs cannot be ruled out. Furthermore, we should still consider the possibility that both drugs contributed or that the combined synergistic effect of the two drugs caused this complication.

In conclusion, although neither everolimus nor polmacoxib has been reported to cause perforation of the colon, the risk of colon perforation should be considered in patients using these medications.

\section{CONFLICTS OF INTEREST}

No potential conflict of interest relevant to this article was reported. 


\section{Coloproctology in-Gyu Song, et al.}

\section{REFERENCES}

1. Baselga J, Campone M, Piccart M, Burris HA 3rd, Rugo HS, Sahmoud T, et al. Everolimus in postmenopausal hormone-receptorpositive advanced breast cancer. N Engl J Med 2012;366:520-9.

2. Paplomata E, Zelnak A, O'Regan R. Everolimus: side effect profile and management of toxicities in breast cancer. Breast Cancer Res Treat 2013;140:453-62.

3. Lee M, Yoo J, Kim JG, Kyung HS, Bin SI, Kang SB, et al. A randomized, multicenter, phase III trial to evaluate the efficacy and safety of polmacoxib compared with celecoxib and placebo for patients with osteoarthritis. Clin Orthop Surg 2017;9:439-57.

4. Aapro M, Andre F, Blackwell K, Calvo E, Jahanzeb M, Papazisis K, et al. Adverse event management in patients with advanced cancer receiving oral everolimus: focus on breast cancer. Ann Oncol 2014;25:763-73.

5. Mychaluk J, Piprot C, Sevestre H, Merviel P, Gondry J, Fauvet R. Intestinal perforation: an unusual adverse event of bevacizumab and paclitaxel combined therapy for a metastatic breast cancer. Presse Med 2009;38:1370-4.
6. Hugl B, Lhotta K, Ensinger C, Cont M, Kafka R, Margreiter R, et al. Colonic perforation associated with leukocytoclastic vasculitis caused by Sirolimus toxicity following renal transplantation. Transpl Int 2006;19:430-1.

7. Fiorentino DF. Cutaneous vasculitis. J Am Acad Dermatol 2003; 48:311-40.

8. Hardinger KL, Cornelius LA, Trulock EP 3rd, Brennan DC. Sirolimus-induced leukocytoclastic vasculitis. Transplantation 2002; 74:739-43.

9. Pasqualotto AC, Bianco PD, Sukiennik TC, Furian R, Garcia VD. Sirolimus-induced leukocytoclastic vasculitis: the second case reported. Am J Transplant 2004;4:1549-51.

10. Nagarajan S, Friedrich T, Garcia M, Kambham N, Sarwal MM. Gastrointestinal leukocytoclastic vasculitis: an adverse effect of sirolimus. Pediatr Transplant 2005;9:97-100.

11. Kim D, Sung YK. New COX-2 inhibitors. Korean J Med 2016;91: 250-6.

12. Thiéfin G, Beaugerie L. Toxic effects of nonsteroidal antiinflammatory drugs on the small bowel, colon, and rectum. Joint Bone Spine 2005;72:286-94. 